\title{
2 Phosphate Recovery by a Surface-Immobilized 3 Cerium Affinity Peptide
}

4 Zihang Su, ${ }^{\dagger}$ Jacob D. Hostert, ${ }^{\dagger}$ Julie N. Renner ${ }^{\dagger}, *$

$5 †$ Department of Chemical and Biomolecular Engineering, Case Western Reserve

6 University, Cleveland, Ohio 44106, United States

$7 \quad *$ Corresponding author: jxr484@case.edu

8 Contents: 12 figures and 3 tables in 24 pages.

9

10

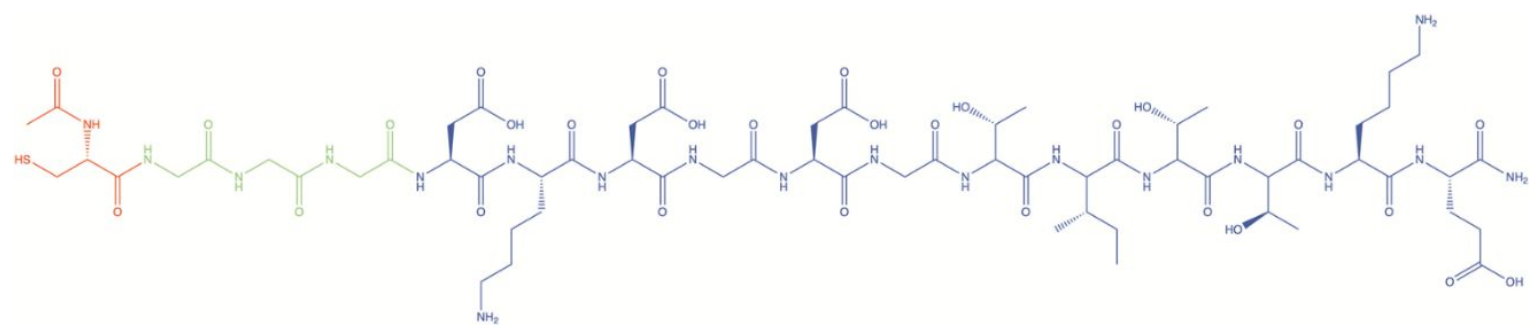

11 Figure S1. Peptide sequence selected for this study. The sequence features cysteine (red)

12 for gold binding via thiol, a spacer (green) to allow for peptide folding, and a calmodulin-

13 derived sequence (blue) for cerium ion binding. 


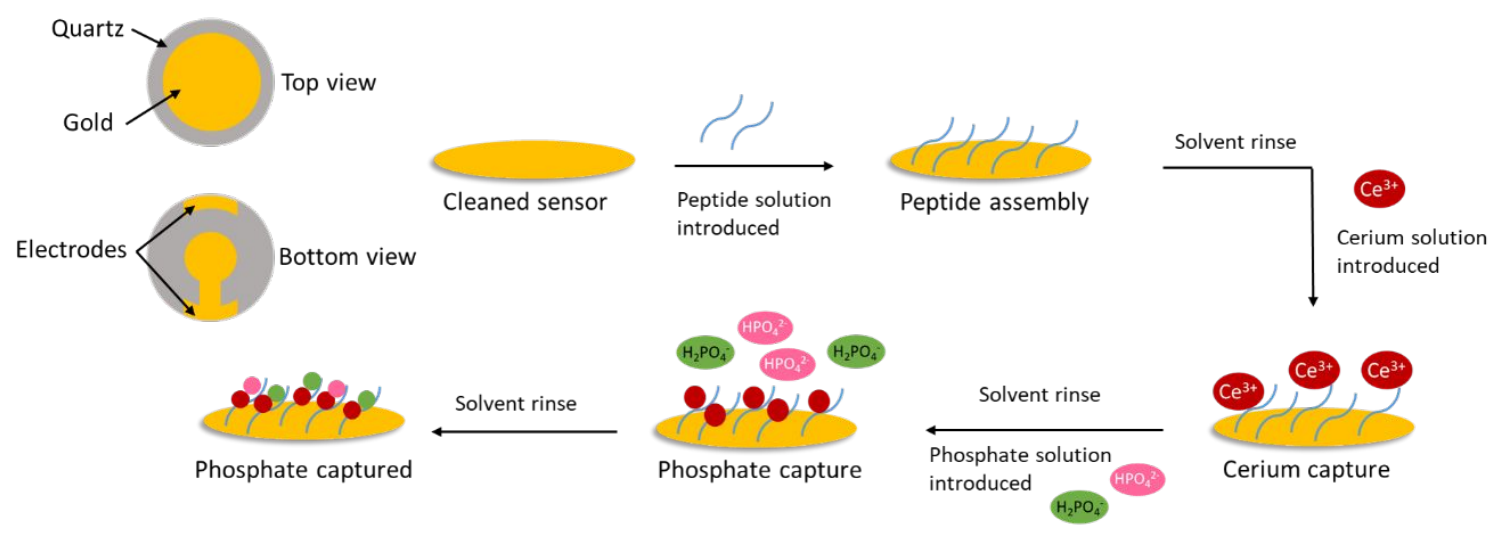

17 Figure S2. A step-by-step description of the peptide-cerium film formation on a gold-

18 coated QCM-D sensor, and the capture of ions by the film. Phosphate is shown as an

19 example. Steps include: 1) peptide assembly followed by solvent rinse, 2) cerium binding

20 followed by solvent rinse, and 3) phosphate ion capture followed by solvent rinse

21

22

23

24

25 
A.

26

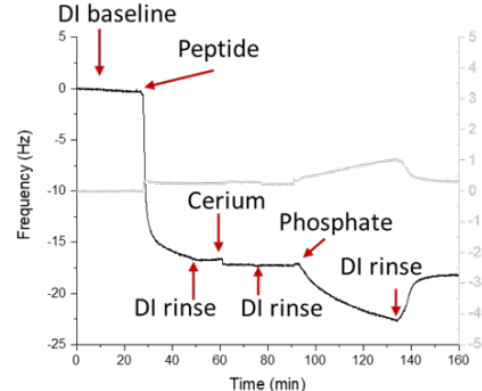

B.

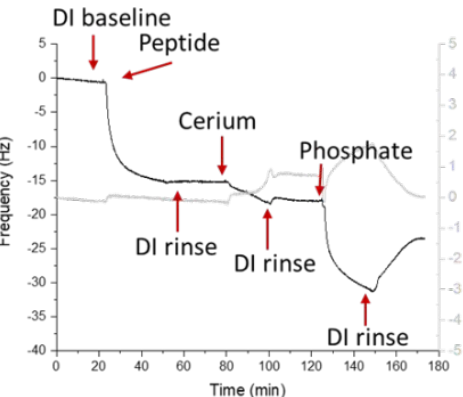

C.

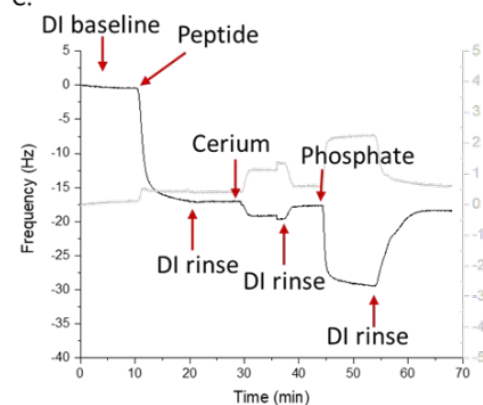

27 Figure S3. QCM-D monitoring of relative frequency shifts (black) and dissipation changes

28 (grey) versus time for peptide adsorption (at $10 \mu \mathrm{g} / \mathrm{mL}$ ), cerium binding (at $1 \mathrm{mM} \mathrm{CeCl}{ }_{3}$ ),

29 and phosphate capture (at $1 \mathrm{mM} \mathrm{Na}_{2} \mathrm{HPO}_{4}$ ) in repeat experiments (A-C) of the data shown

30 in Figure 1 of the main text. Red arrows indicate when different solutions were introduced

31 to the flow module.

32

33

34

35

36

37 
A.

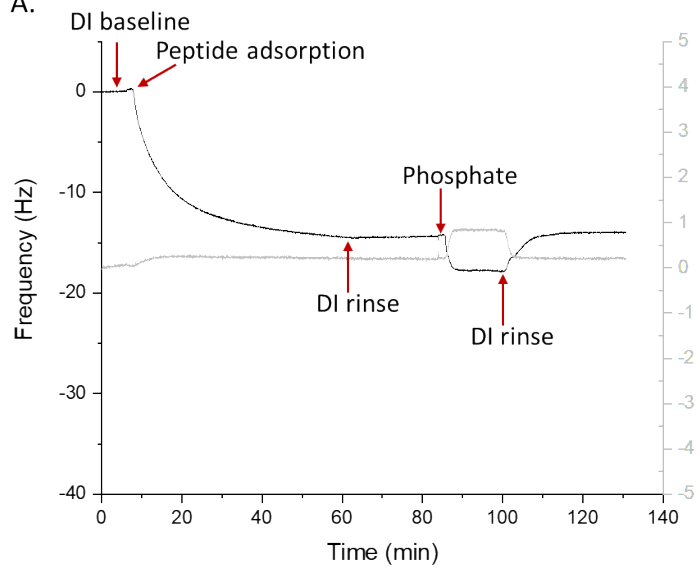

B.

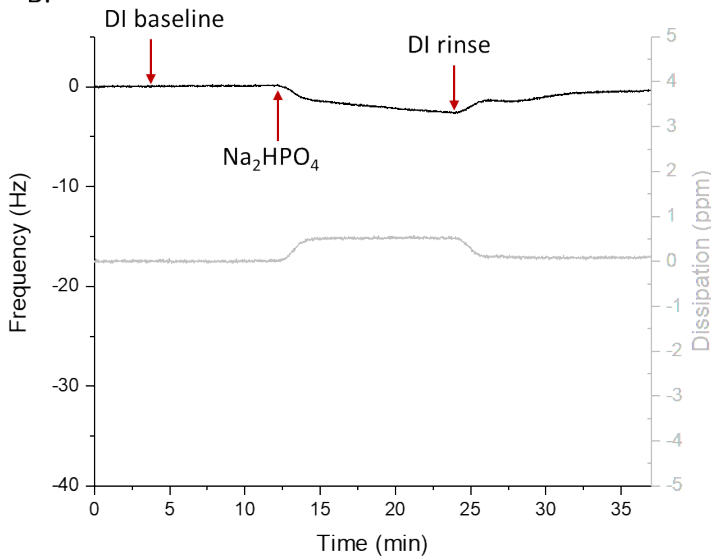

39

40 Figure S4. QCM-D monitoring of frequency shift (black) and dissipation changes (grey)

41 versus time of (A) the formation of a stable peptide film and subsequent exposure to

42 phosphate $\left(10 \mathrm{mM} \mathrm{Na}_{2} \mathrm{HPO}_{4} \mathrm{pH}\right.$ 9.1) followed by a DI water rinse, and (B) a $7 \mathrm{mM}$

$43 \mathrm{Na}_{2} \mathrm{HPO}_{4}$ solution introduced to bare gold coated sensors. Higher concentrations than the

44 experiments in Figure 1 of the manuscript were selected for control experiments. Red

45 arrows indicate when different solutions were introduced.

47

48

49 

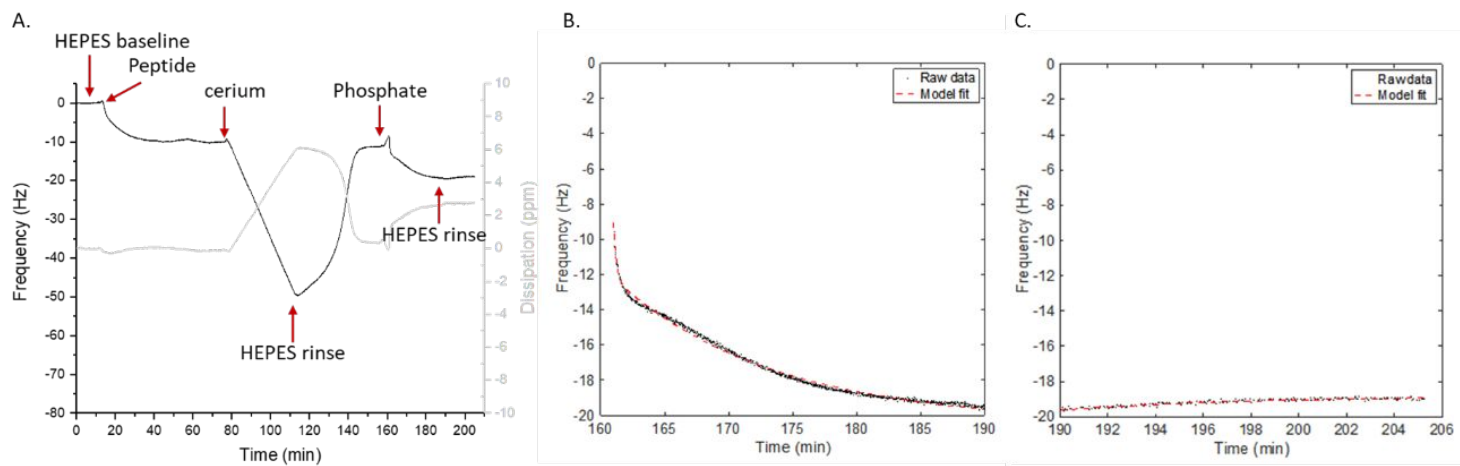

50

51 Figure S5. QCM-D monitoring of frequency shift (black) and dissipation changes (grey)

52 versus time of (A) the formation of a stable peptide-cerium film and subsequent exposure

53 to phosphate $\left(1 \mathrm{mM} \mathrm{Na}_{2} \mathrm{HPO}_{4}\right)$ followed by rinse step all in HEPES buffer (pH 7.2). Red

54 arrows indicate the solution changes. (B) Modeling of phosphate adsorption data on a

55 cerium-peptide immobilized film in HEPES buffer. (C) Modeling of phosphate desorption

56 data during the final rinse with HEPES buffer. Red dashed lines represent results of

57 nonlinear least squares curve fitting in B and C, performed using MATLAB ${ }^{\circledR}$.

58

59

60 

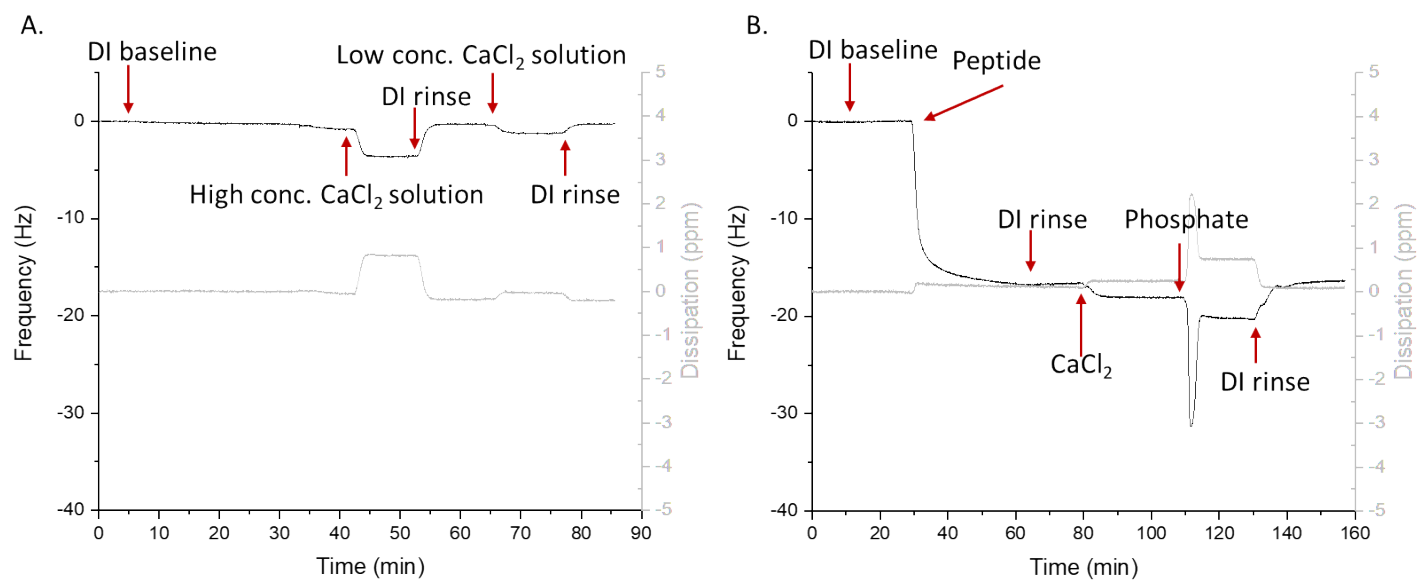

61

62 Figure S6. QCM-D monitoring of relative frequency (black) and dissipation (grey) changes

63 versus time for $(\mathrm{A})$ solutions of $\mathrm{CaCl}_{2}(1 \mathrm{mM}$ and $1 \mathrm{M})$ introduced to a bare gold-coated

64 crystal sensor surface followed by DI rinse, and (B) solutions of $\mathrm{CaCl}_{2}(1 \mathrm{mM})$ introduced

65 to a peptide-functionalized gold-coated sensor followed by phosphate exposure (in form of

$661 \mathrm{mM} \mathrm{Na}_{2} \mathrm{HPO}_{4}, \mathrm{pH}$ 9.2). Red arrows indicate when new solutions were introduced to the

67 sensor surface.

68

69 


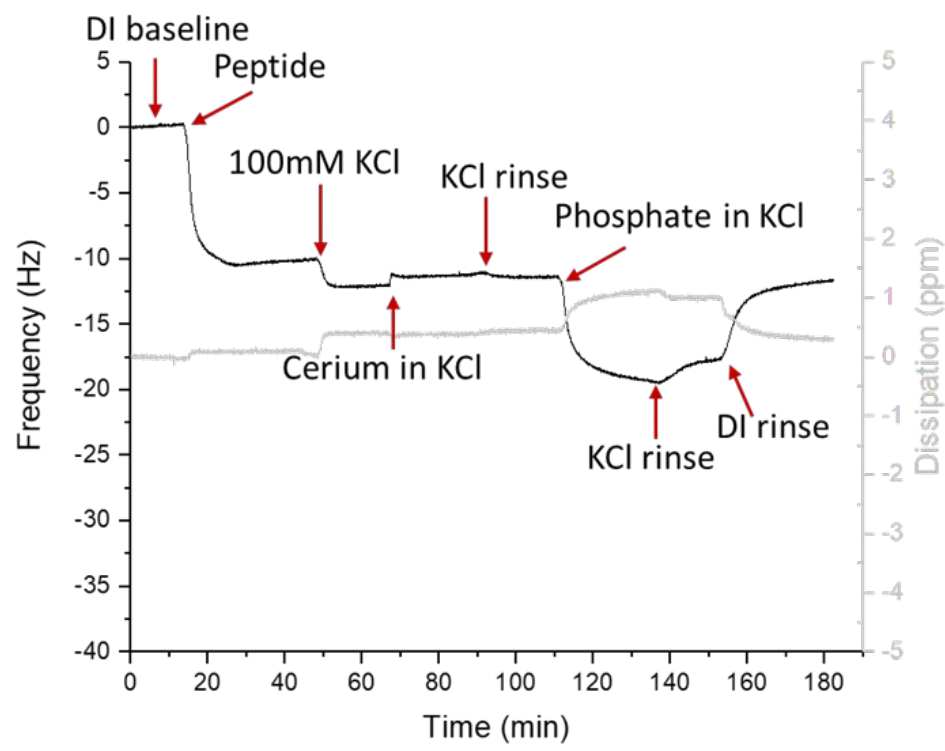

70

71 Figure S7. QCM-D monitoring of relative frequency shifts (black) and dissipation changes

72 (grey) versus time for peptide films exposed to $100 \mathrm{mM} \mathrm{KCl}$ solution, followed by cerium

73 binding $\left(1 \mathrm{mM} \mathrm{CeCl}_{3}\right)$ and phosphate $\left(1 \mathrm{mM} \mathrm{Na}_{2} \mathrm{HPO}_{4}\right)$ capture in $100 \mathrm{mM} \mathrm{KCl}$ solution,

74 followed by a $100 \mathrm{mM} \mathrm{KCl} \mathrm{rinse,} \mathrm{and} \mathrm{a} \mathrm{final} \mathrm{DI} \mathrm{water} \mathrm{rinse.} \mathrm{Red} \mathrm{arrows} \mathrm{indicate} \mathrm{when} \mathrm{a}$

75 new solution was introduced to the sensor surface.

76

77 
A.

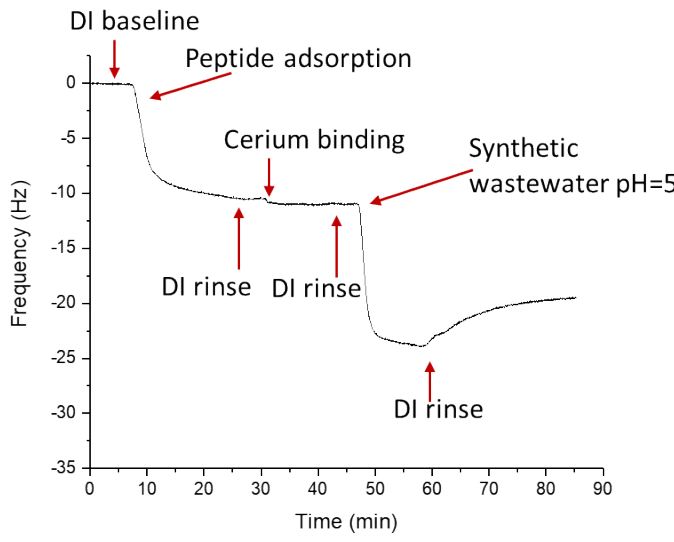

B.

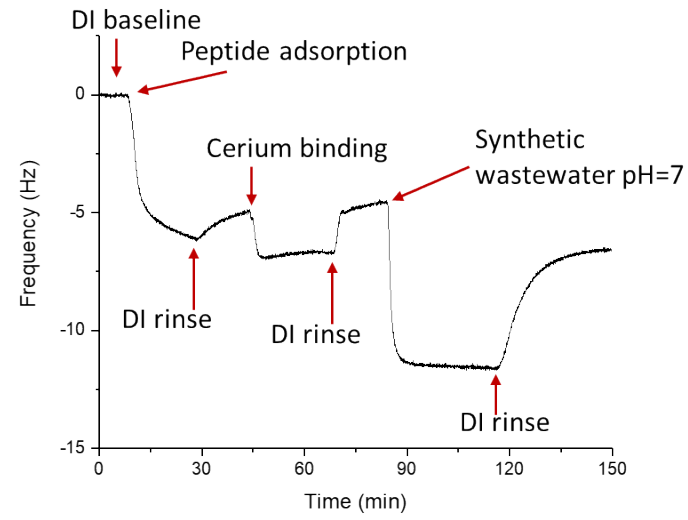

79 Figure S8. Repeat QCM-D monitoring of relative frequency (black) and dissipation (grey)

80 changes versus time for (A) peptide-cerium films exposed to synthetic wastewater ( $\mathrm{pH} 5$ ),

81 and (B) peptide-cerium films exposed to synthetic wastewater ( $\mathrm{pH}$ 7). DI water served as

82 the baseline and rinse medium. Red arrows indicate when a new solution was introduced to

83 the sensor surface.

84

85

86 

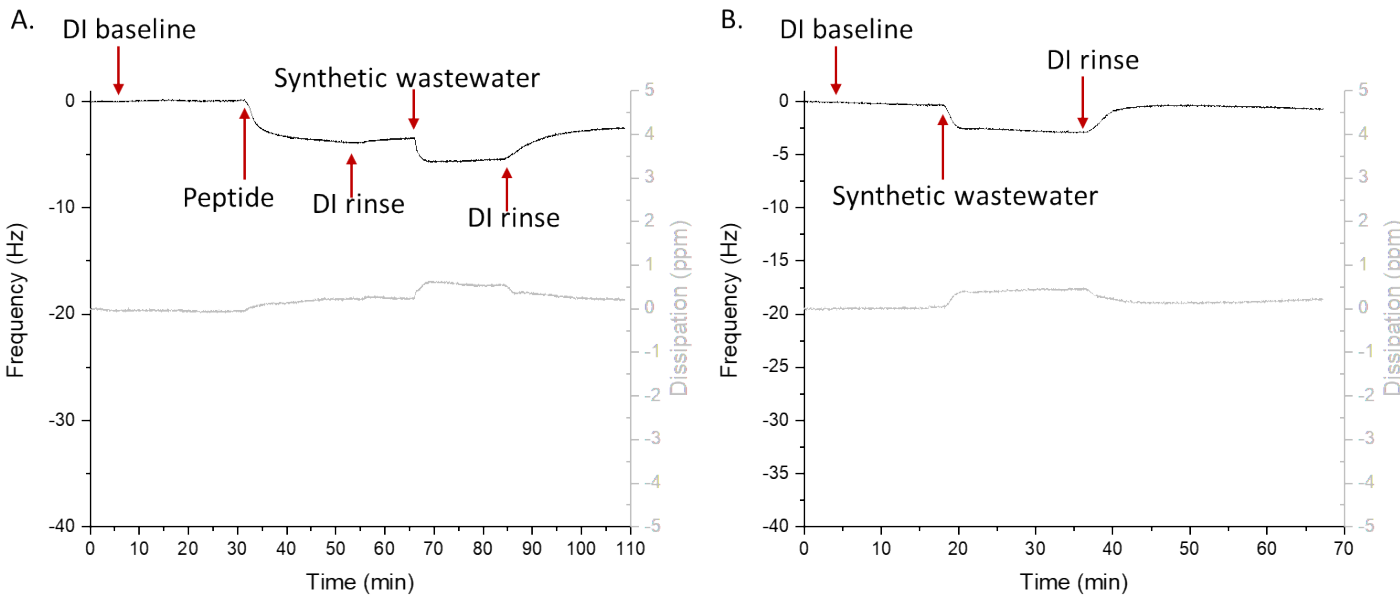

87

88 Figure S9. QCM-D monitoring of relative frequency (black) and dissipation (grey) changes

89 versus time for (A) the peptide exposed to synthetic wastewater ( $\mathrm{pH} 7)$, and (B) the bare

90 gold exposed to synthetic wastewater $(\mathrm{pH} 7)$. Red arrows indicate when a new solution was

91 introduced to the sensor surface.

92

93

94

95 
A.

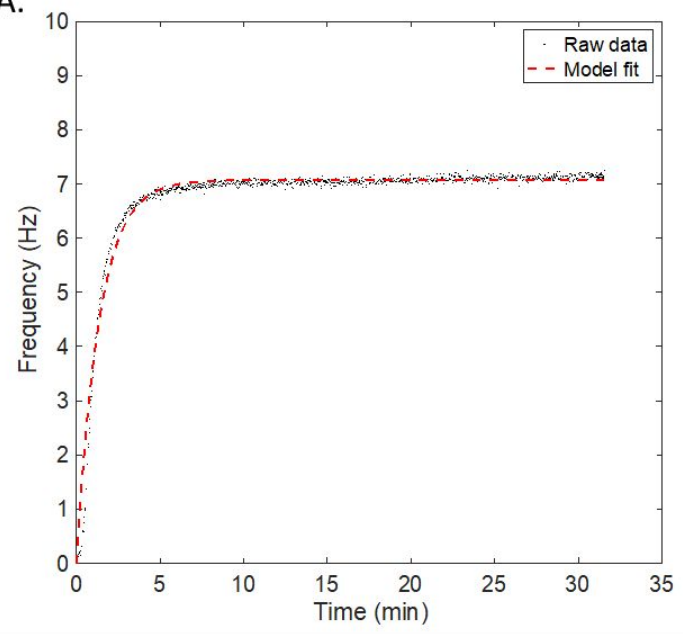

B.

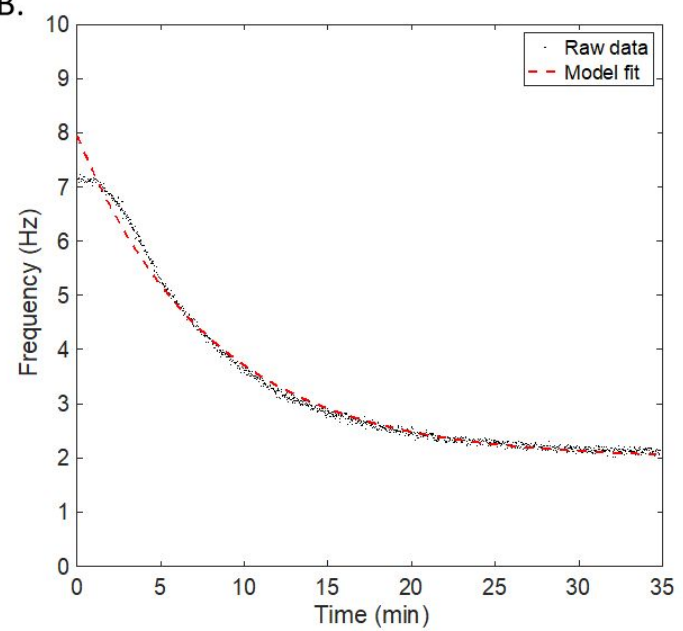

97 Figure S10. Modeling synthetic wastewater adsorption and desorption data at $\mathrm{pH}$ 7. (A)

98 Modeling of phosphate adsorption data on the cerium-peptide immobilized film according

99 to Note $1, \mathrm{R}^{2}=0.996$ (B) Modeling of phosphate desorption data during the final DI water

100 rinse process according to Note $2, \mathrm{R}^{2}=0.991$. Red dashed lines represent results of nonlinear

101 least squares curve fitting in $\mathrm{A}$ and $\mathrm{B}$, performed using MATLAB ${ }^{\circledR}$.

102

103

104 
A.

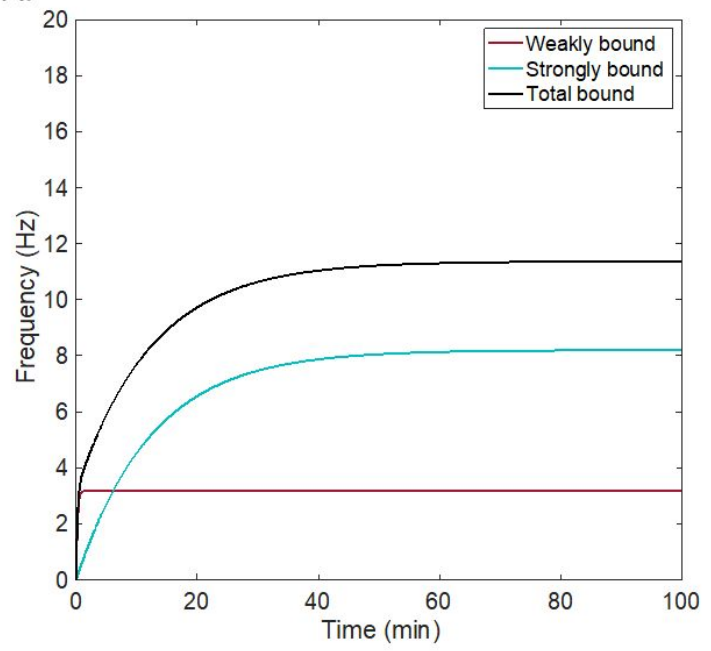

B.

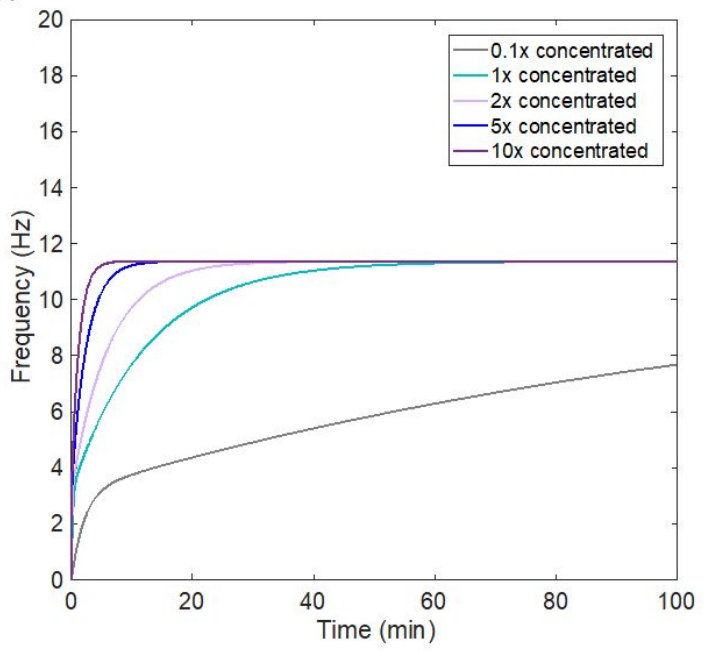

106 Figure S11: Modeling of phosphate adsorption to peptide-cerium films in synthetic

107 wastewater. (A) Synthetic system profile in HEPES at $\mathrm{pH} 7$ using fitting parameters obtained

108 via nonlinear regression per Note 1. Parameters used are: $\mathrm{k}_{\mathrm{t}} \mathrm{A}=0.081 \mathrm{~s}^{-1}, \mathrm{k}_{\mathrm{a}} \mathrm{A}=4.7 \mathrm{~s}^{-1}$, and

$109 \mathrm{k}_{\mathrm{d}}=0.0028 \mathrm{~s}^{-1}$ The red line corresponds to the frequency shift due to weakly bound ions,

110 while the teal line corresponds to the frequency shift of strongly bound ions. The black line

111 is the sum of the weakly and strongly bound ions, and corresponds to what is actually

112 measured. (B) The synthetic impact of increasing concentration on the total phosphate bound

113 with time using parameters obtained from fitting data at in HEPES at $\mathrm{pH}$ 7. 1X concentrated

114 refers to $1 \mathrm{mM}$. 


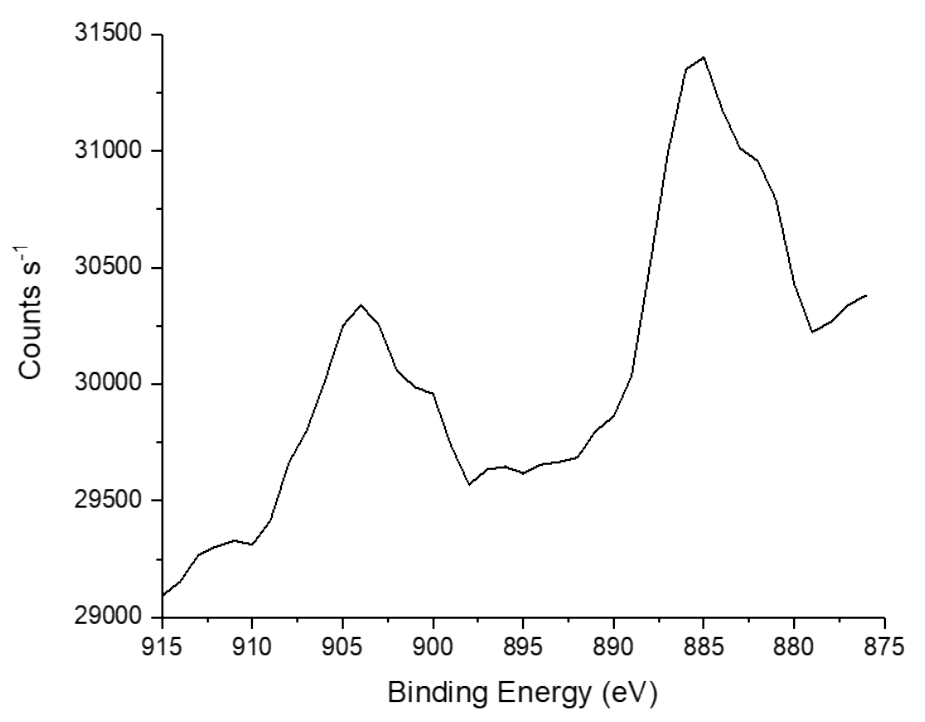

116

117 Figure S12. XPS spectrum of Ce 3d on gold-coated crystal sensor sample with a peptide-

118 cerium film assembled via QCM-D. The Ce 3d spectrum displays two bands at $904 \mathrm{eV}$ and $119885 \mathrm{eV}$, confirming the cerium was adsorbed by peptide.

120

121

122

123

124

125

126

127 
128 Table S1: The average adjusted $\mathrm{R}^{2}$ values for various model fits on data from Figure 1 and 129 repeats in Figure $\mathbf{S 3}$ with $\mathrm{n}=4$. The note refers to the derivation of the model used for 130 fitting. The first order model for desorption is equivalent to the derived model.

Adjusted $\mathrm{R}^{2}$

\begin{tabular}{cccccc}
\hline First order (Note 4) & \multicolumn{2}{c}{ Second order (Note 5) } & \multicolumn{2}{c}{ Derived model } \\
& & & & \\
& & & \\
& & & & \\
& & & & \\
\hline Adsotes 1 and 2) & \\
\hline 0.975 & - & 0.716 & 0.808 & 0.998 & 0.972 \\
0.026 & - & 0.156 & 0.117 & 0.001 & 0.021 \\
\hline
\end{tabular}

131

132 Table S2: The adjusted $\mathrm{R}^{2}$ values for control experiments shown in Figure $\mathbf{S 4}$ with $\mathrm{n}=1$

133 for each control. The note refers to the derivation used for fitting. The first order model

134 for desorption and adsoprtion is equivalent to the derived model.

Adjusted R ${ }^{2}$

\begin{tabular}{lcccccc}
\cline { 2 - 6 } & \multicolumn{2}{c}{ First order (Note 5) } & \multicolumn{2}{c}{ Second order (Note 4) } & \multicolumn{2}{c}{ Derived model (Note 3) } \\
\cline { 2 - 6 } & Adsorption & Desorption & Adsorption & Desorption & Adsorption & Desorption \\
\cline { 2 - 7 } $\begin{array}{l}\text { Peptide } \\
\text { only }\end{array}$ & - & - & 0.004 & 0.624 & 0.988 & 0.990 \\
Bare gold & - & - & 0.007 & 0.860 & 0.974 & 0.940 \\
\hline
\end{tabular}


136 Table S3. Summary of the kinetic constants obtained by fitting Equation 1 to QCM-D 137 adsorption data obtained when exposing stable peptide-cerium films to phosphate in 138 HEPES buffer at $\mathrm{pH} 7$ and to $\mathrm{pH} 7$ synthetic wastewater (SWW). For HEPES data, $\mathrm{n}=1$. 139 For SWW data, $\mathrm{n}=2$. Nonlinear least squares curve fitting was performed using $140 \operatorname{MATLAB}^{\circledR}$. For pH 7, A = $1 \mathrm{mM}$. For SWW pH 7, A = 2 mM.

\section{pH 7 SWW pH 7}

$\begin{array}{rrr}\mathrm{k}_{\mathrm{a}} \mathrm{A}\left(\mathrm{s}^{-1}\right) & 4.7 & 2.1 \\ \mathrm{k}_{\mathrm{d}}\left(\mathrm{s}^{-1}\right) & 0.0027 & 0.053 \\ \mathrm{k}_{\mathrm{t}} \mathrm{A}\left(\mathrm{s}^{-1}\right) & 0.081 & 0.13\end{array}$

141

142

143

144

145

146

147 
148 Note 1: Derivation of phosphate adsorption to peptide-cerium films

149 Assume the system has two parallel steps:

150

$$
\mathrm{A}+\mathrm{B} \rightleftharpoons \mathrm{AB}{ }^{*}
$$

151

$$
\mathrm{A}+\mathrm{B} \rightarrow \mathrm{AB}
$$

152 Generally, $\mathrm{A}$ is the species adsorbing (phosphate) and B is the adsorber (the adsorption

153 site). The first equilibrium step represents weak interactions (with $\mathrm{k}_{\mathrm{a}}$ describing forward

154 reaction rate constant and $\mathrm{k}_{\mathrm{d}}$ describing the reverse reaction rate constant). The second

155 reaction is the strong binding step, with rate constant $\mathrm{k}_{\mathrm{t}}$. See equation 19 for the final form.

156 Write out the rate equations:

157

1: $\frac{\mathrm{dAB}}{\mathrm{dt}}=\mathrm{k}_{\mathrm{a}} * \mathrm{~A} * \mathrm{~B}^{*}-\mathrm{k}_{\mathrm{d}} \mathrm{AB}{ }^{*}$

158

2: $\frac{\mathrm{dAB}}{\mathrm{dt}}=\mathrm{k}_{\mathrm{t}} * \mathrm{~A} * \mathrm{~B}$

159 The number of sites is constant for both steps:

160

3: $\mathrm{B}^{*}+\mathrm{AB}^{*}=\mathrm{B}_{0}{ }^{*}$

161

4: $\quad \mathrm{B}+\mathrm{AB}=\mathrm{B}_{0}$

162 Substitute into the rate equations to eliminate B.

163

5: $\frac{\mathrm{dAB}^{*}}{\mathrm{dt}}=\mathrm{k}_{\mathrm{a}} * \mathrm{~A} *\left(\mathrm{~B}_{0}^{*}-\mathrm{AB}^{*}\right)-\mathrm{k}_{\mathrm{d}} \mathrm{AB} *$ 
165 Solve equation 1.

166

$$
\text { 7: } \frac{\mathrm{dAB}^{*}}{\mathrm{dt}}+\left(\mathrm{k}_{\mathrm{a}} * \mathrm{~A}+\mathrm{k}_{\mathrm{d}}\right) * \mathrm{AB} *=\mathrm{k}_{\mathrm{a}} * \mathrm{~A} * \mathrm{~B}_{0} *
$$

167 Introduce an integrating factor:

168 8: $\quad$ IF $=\mathrm{e}^{\int\left(\mathrm{k}_{\mathrm{a}} * \mathrm{~A}+\mathrm{k}_{\mathrm{d}}\right) \mathrm{dt}}=\mathrm{e}^{\left(\mathrm{k}_{\mathrm{a}} * \mathrm{~A}+\mathrm{k}_{\mathrm{d}}\right) \mathrm{t}}$

169

9: $\int\left[\mathrm{AB}^{*} * \mathrm{e}^{\left(\mathrm{k}_{\mathrm{a}} * \mathrm{~A}+\mathrm{k}_{\mathrm{d}}\right) \mathrm{t}}\right]^{\prime}=\mathrm{k}_{\mathrm{a}} * \mathrm{~A} * \mathrm{~B}_{0} * \int \mathrm{e}^{\left(\mathrm{k}_{\mathrm{a}} * \mathrm{~A}+\mathrm{k}_{\mathrm{d}}\right) \mathrm{t}} \mathrm{dt}$

170

10: $\mathrm{AB}^{*} * \mathrm{e}^{\left(\mathrm{k}_{\mathrm{a}} * \mathrm{~A}+\mathrm{k}_{\mathrm{d}}\right) \mathrm{t}}=\frac{\mathrm{k}_{\mathrm{a}} * \mathrm{~A} * \mathrm{~B}_{0}{ }^{*}}{\mathrm{k}_{\mathrm{a}} * \mathrm{~A}+\mathrm{k}_{\mathrm{d}}} \mathrm{e}^{\left(\mathrm{k}_{\mathrm{a}} * \mathrm{~A}+\mathrm{k}_{\mathrm{d}}\right) \mathrm{t}}+\mathrm{C}$

171 At $\mathrm{t}=0, \mathrm{AB}^{*}=0$

172 11: $0=\frac{\mathrm{k}_{\mathrm{a}} * \mathrm{~A} * \mathrm{~B}_{0}}{\mathrm{k}_{\mathrm{a}} * \mathrm{~A}+\mathrm{k}_{\mathrm{d}}}+C$

173 12: $\mathrm{C}=-\frac{\mathrm{k}_{\mathrm{a}} * \mathrm{~A} * \mathrm{~B}_{0}{ }^{*}}{\mathrm{k}_{\mathrm{a}} * \mathrm{~A}+\mathrm{k}_{\mathrm{d}}}$

174 Divide by the exponential on the LHS

175

13: $\mathrm{AB}^{*}=\frac{\mathrm{k}_{\mathrm{a}} * \mathrm{~A} * \mathrm{~B}_{0}{ }^{*}}{\mathrm{k}_{\mathrm{a}} * \mathrm{~A}+\mathrm{k}_{\mathrm{d}}}-\frac{\mathrm{k}_{\mathrm{a}} * \mathrm{~A} * \mathrm{~B}_{0}{ }^{*}}{\mathrm{k}_{\mathrm{a}} * \mathrm{~A}+\mathrm{k}_{\mathrm{d}}} \mathrm{e}^{-\left(\mathrm{k}_{\mathrm{a}} * \mathrm{~A}+\mathrm{k}_{\mathrm{d}}\right) \mathrm{t}}$

176

14: $\mathrm{AB}^{*}=\frac{\mathrm{k}_{\mathrm{a}} * \mathrm{~A} * \mathrm{~B}_{0}{ }^{*}}{\mathrm{k}_{\mathrm{a}} * \mathrm{~A}+\mathrm{k}_{\mathrm{d}}}\left(1-\mathrm{e}^{-\left(\mathrm{k}_{\mathrm{a}} * \mathrm{~A}+\mathrm{k}_{\mathrm{d}}\right) \mathrm{t}}\right)$

177 Solve equation 6:

178 15: $\int_{0}^{\mathrm{AB}} \frac{\mathrm{dAB}}{\left(\mathrm{B}_{0}-\mathrm{AB}\right)}=\mathrm{k}_{\mathrm{t}} * \mathrm{~A} * \int_{0}^{\mathrm{t}} \mathrm{dt}$ 
$179 \quad 16:-\ln \left(\frac{\mathrm{B}_{0}-\mathrm{AB}}{\mathrm{B}_{0}}\right)=\mathrm{k}_{\mathrm{t}} * \mathrm{~A} * \mathrm{t}$

180 Exponentiate and multiply by $\mathrm{B}_{0}$

$181 \quad 17: \mathrm{B}_{0}-\mathrm{AB}=\mathrm{B}_{0} \mathrm{e}^{-\mathrm{k}_{\mathrm{t}} * \mathrm{~A} * \mathrm{t}}$

182 18: $\mathrm{AB}=\mathrm{B}_{0}\left(1-\mathrm{e}^{-\mathrm{k}_{\mathrm{t}} * \mathrm{~A} * \mathrm{t}}\right)$

183 We measure $\mathrm{AB}+\mathrm{AB}$ *

184 19: $\mathrm{AB}+\mathrm{AB}^{*}=\overline{\mathrm{AB}}=\mathrm{B}_{0}\left(1-\mathrm{e}^{-\mathrm{k}_{\mathrm{t}} * \mathrm{~A} * \mathrm{t}}\right)+\frac{\mathrm{k}_{\mathrm{a}} * \mathrm{~A} * \mathrm{~B}_{0}{ }^{*}}{\mathrm{k}_{\mathrm{a}} * \mathrm{~A}+\mathrm{k}_{\mathrm{d}}}\left(1-\mathrm{e}^{-\left(\mathrm{k}_{\mathrm{a}} * \mathrm{~A}+\mathrm{k}_{\mathrm{d}}\right) \mathrm{t}}\right)$

185

186

187

188

189

190

191

192

193 
195 During the desorption period, A->0. See equation 27 for the final form.

$19620: \frac{\mathrm{dAB}^{*}}{\mathrm{dt}}=-\mathrm{k}_{\mathrm{d}} \mathrm{AB}{ }^{*}$

$19721: \frac{\mathrm{dAB}}{\mathrm{dt}}=0$

198 Integrate equation 6 : where $\mathrm{F}^{*}{ }_{0}$ is the initial frequency due to weak binding

$19922: \int_{\mathrm{F}^{*}{ }_{0}}^{\mathrm{AB}^{*}} \frac{\mathrm{dAB}^{*}}{\mathrm{AB}^{*}}=-\mathrm{k}_{\mathrm{d}} \int_{0}^{\mathrm{t}} \mathrm{dt}$

$20023: \ln \left(\frac{\mathrm{AB}^{*}}{\mathrm{~F}^{*}{ }_{0}}\right)=-\mathrm{k}_{\mathrm{d}} \mathrm{t}$

$20124: \mathrm{AB}^{*}=\mathrm{F}^{*}{ }_{0} \mathrm{e}^{-\mathrm{k}_{\mathrm{d}} \mathrm{t}}$

202 Integrate equation 7: where $\mathrm{F}_{0}$ is the initial frequency due to strong binding

$20325: \int_{\mathrm{F}_{0}}^{\mathrm{AB}} \mathrm{dAB}=0$

$20426: \quad \mathrm{AB}=\mathrm{F}_{0}$

205 We measure $\mathrm{AB}+\mathrm{AB} *$

206

27: $\mathrm{AB}+\mathrm{AB}^{*}=\overline{\mathrm{AB}}=\mathrm{F}_{0}+\mathrm{F}^{*}{ }_{0} \mathrm{e}^{-\mathrm{k}_{\mathrm{d}} \mathrm{t}}$

207

208 
only

211 Assume the system has one step:

$$
\mathrm{A}+\mathrm{B} \rightleftharpoons \mathrm{AB} *
$$

213 Generally, $\mathrm{A}$ is the species adsorbing and $\mathrm{B}$ is the adsorber. The equilibrium step

214 represents weak electrostatic interactions (with $\mathrm{k}_{\mathrm{a}}$ describing forward reaction rate constant

215 and $k_{d}$ describing the reverse reaction rate constant). See equations 39 and 45 for the final

216 forms of adsorption and desorption respectively.

$21728: \frac{\mathrm{dAB}^{*}}{\mathrm{dt}}=\mathrm{k}_{\mathrm{a}} \mathrm{B} * \mathrm{~A}-\mathrm{k}_{\mathrm{d}} \mathrm{AB}{ }^{*}$

218 Assume the total number of sites is constant

219

29: $\mathrm{B}^{*}+\mathrm{AB}^{*}=\mathrm{B}_{0}^{*}$

220 Substitute in to replace B*

$221 \quad 30: \frac{\mathrm{dAB}^{*}}{\mathrm{dt}}=\mathrm{k}_{\mathrm{a}}\left(\mathrm{B}_{0}{ }^{*}-\mathrm{AB}^{*}\right) * \mathrm{~A}-\mathrm{k}_{\mathrm{d}} \mathrm{AB}{ }^{*}$

222 Distribute and rearrange:

223

31: $\frac{\mathrm{dAB}^{*}}{\mathrm{dt}}+\left(\mathrm{k}_{\mathrm{a}} * \mathrm{~A}+\mathrm{k}_{\mathrm{d}}\right) * \mathrm{AB}{ }^{*}=\mathrm{k}_{\mathrm{a}} * \mathrm{~A} * \mathrm{~B}_{0} *$

224 Introduce an integrating factor 
225 32: $I F=\mathrm{e}^{\int\left(\mathrm{k}_{\mathrm{a}} * \mathrm{~A}+\mathrm{k}_{\mathrm{d}}\right) \mathrm{dt}}=\mathrm{e}^{\left(\mathrm{k}_{\mathrm{a}} * \mathrm{~A}+\mathrm{k}_{\mathrm{d}}\right) \mathrm{t}}$

226 33: $\left[A B * * \mathrm{e}^{\left(\mathrm{k}_{\mathrm{a}} * \mathrm{~A}+\mathrm{k}_{\mathrm{d}}\right) \mathrm{t}}\right]^{\prime}=\mathrm{k}_{\mathrm{a}} * \mathrm{~A} * \mathrm{~B}_{0} * * \mathrm{e}^{\left(\mathrm{k}_{\mathrm{a}} * \mathrm{~A}+\mathrm{k}_{\mathrm{d}}\right) \mathrm{t}}$

227 Integrate:

$22834: \mathrm{AB}^{*} * \mathrm{e}^{\left(\mathrm{k}_{\mathrm{a}} * \mathrm{~A}+\mathrm{k}_{\mathrm{d}}\right) \mathrm{t}}=\mathrm{k}_{\mathrm{a}} * \mathrm{~A} * \mathrm{~B}_{0} * \int \mathrm{e}^{\left(\mathrm{k}_{\mathrm{a}} * \mathrm{~A}+\mathrm{k}_{\mathrm{d}}\right) \mathrm{t}} \mathrm{dt}$

$22935: \mathrm{AB}^{*} * \mathrm{e}^{\left(\mathrm{k}_{\mathrm{a}} * \mathrm{~A}+\mathrm{k}_{\mathrm{d}}\right) \mathrm{t}}=\frac{\mathrm{k}_{\mathrm{a}} * \mathrm{~A} * \mathrm{~B}_{0}{ }^{*}}{\mathrm{k}_{\mathrm{a}} * \mathrm{~A}+\mathrm{k}_{\mathrm{d}}} * \mathrm{e}^{\left(\mathrm{k}_{\mathrm{a}} * \mathrm{~A}+\mathrm{k}_{\mathrm{d}}\right) \mathrm{t}}+\mathrm{C}$

230 Where $\mathrm{C}$ is an integration constant.

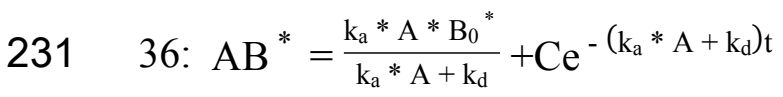

232 Solve for the integration constant by setting the initial condition at $\mathrm{t}=0$, there is no surface

233 coverage

234 37: $0=\frac{\mathrm{k}_{\mathrm{a}} * \mathrm{~A} * \mathrm{~B}_{0}{ }^{*}}{\mathrm{k}_{\mathrm{a}} * \mathrm{~A}+\mathrm{k}_{\mathrm{d}}}+\mathrm{C}$

235 38: $\mathrm{C}=\frac{-\mathrm{k}_{\mathrm{a}} * \mathrm{~A} * \mathrm{~B}_{0}{ }^{*}}{\mathrm{k}_{\mathrm{a}} * \mathrm{~A}+\mathrm{k}_{\mathrm{d}}}$

236 Finally, arrive at

237 39: $A B^{*}=\frac{\mathrm{k}_{\mathrm{a}}^{*} \mathrm{~A} * \mathrm{~B}_{0}{ }^{*}}{\mathrm{k}_{\mathrm{a}} * \mathrm{~A}+\mathrm{k}_{\mathrm{d}}}\left(1-\mathrm{e}^{-\left(\mathrm{k}_{\mathrm{a}} * \mathrm{~A}+\mathrm{k}_{\mathrm{d}}\right) \mathrm{t}}\right)$

238 For the desorption period, $\mathrm{A}=0$

239

40: $\frac{\mathrm{dAB}^{*}}{\mathrm{dt}}=-\mathrm{k}_{\mathrm{d}} \mathrm{AB}{ }^{*}$ 
240 41: $\int \frac{\mathrm{dAB}^{*}}{\mathrm{AB}^{*}}=-\mathrm{k}_{\mathrm{d}} \int \mathrm{dt}$

$24142: \ln \left(\mathrm{AB}^{*}\right)=-\mathrm{k}_{\mathrm{d}} \mathrm{t}+\mathrm{C}$

242 43: $\mathrm{AB}^{*}=\check{\mathrm{C}} \mathrm{e}^{-\mathrm{k}_{\mathrm{d}} \mathrm{t}}$

243 At $\mathrm{t}=0$, there is an initial frequency $\mathrm{F}_{0}$

244 44: $\mathrm{F}_{0}=\check{\mathrm{C}}$

245 Finally,

$24645: \mathrm{AB}^{*}=\mathrm{F}_{0} \mathrm{e}^{-\mathrm{k}_{\mathrm{d}} \mathrm{t}}$

247

248

249

250

251

252

253

254 
256 Starting from the most general rate equation of order $n$. See equations 52 and 55 for the

257 final forms used in adsorption and desorption respectively.

$258 \quad 46: \frac{d q(t)}{d t}=k_{n}\left[q_{e}-q(t)\right]^{n}$

259 Where:

$260 \mathrm{q}(\mathrm{t})=$ amount of adsorbed solute

$261 \mathrm{k}_{\mathrm{n}}=$ the $\mathrm{n}^{\text {th }}$ order rate constant

$262 \mathrm{q}_{\mathrm{e}}=$ equilibrium amount of adsorbed solute

263 Fixing the order at $\mathrm{n}=1$

$264 \quad 47: \frac{d q(t)}{d t}=k_{1}\left[q_{e}-q(t)\right]$

265 Separate the terms and integrate, where $\mathrm{C}$ is an integration constant:

266 48: $\int \frac{\mathrm{dq}(\mathrm{t})}{\left[\mathrm{q}_{\mathrm{e}}-\mathrm{q}(\mathrm{t})\right]}=\int \mathrm{k}_{1} \mathrm{dt}$

267 49: $\ln \left[\mathrm{q}_{\mathrm{e}}-\mathrm{q}(\mathrm{t})\right]=-\mathrm{k}_{1} \mathrm{t}+\mathrm{C}$

268 For adsorption, apply the initial condition $\mathrm{q}(0)=0$

$26950: \ln \left[\mathrm{q}_{\mathrm{e}}\right]=\mathrm{C}$ 
271 51: $\ln \left[\mathrm{q}_{\mathrm{e}}-\mathrm{q}(\mathrm{t})\right]=-\mathrm{k}_{1} \mathrm{t}+\ln \left(\mathrm{q}_{\mathrm{e}}\right)$

272 Or equivalently,

273 52: $\mathrm{q}(\mathrm{t})=\mathrm{q}_{\mathrm{e}}\left(1-\mathrm{e}^{-\mathrm{k}_{1} \mathrm{t}}\right)$

274 For desorption, apply the initial condition $\mathrm{q}(0)=\mathrm{q}_{0}$ to equation 49

275 53: $\ln \left[\mathrm{q}_{\mathrm{e}}-\mathrm{q}_{0}\right]=\mathrm{C}$

276 54: $\ln \left[\mathrm{q}_{\mathrm{e}}-\mathrm{q}(\mathrm{t})\right]=-\mathrm{k}_{1} \mathrm{t}+\ln \left[\mathrm{q}_{\mathrm{e}}-\mathrm{q}_{0}\right]$

277 Or equivalently,

278 55: $\mathrm{q}(\mathrm{t})=\mathrm{q}_{\mathrm{e}}-\left(\mathrm{q}_{\mathrm{e}}-\mathrm{q}_{0}\right) \mathrm{e}^{-\mathrm{k}_{1} \mathrm{t}}$

279

280

281

282

283

284 
286 See equations 60 and 62 for the final forms used in adsorption and desorption respectively

287 Set $\mathrm{n}=2$ on equation 46 .

$288 \quad 56: \frac{d q(t)}{d t}=k_{2}\left[q_{e}-q(t)\right]^{2}$

289 Separate and integrate, $\mathrm{C}$ is an integration constant

$290 \quad 57: \int \frac{d q(t)}{\left[q_{e}-q(t)\right]^{2}}=\int k_{2} d t$

$29158: \frac{-1}{\left[q_{e}-q(t)\right]}=k_{2} t+C$

292 For adsorption, $\mathrm{q}(0)=0$

$293 \quad 59: \frac{-1}{q_{\mathrm{e}}}=\mathrm{C}$

$29460: \frac{-1}{\left[\mathrm{q}_{\mathrm{e}}-\mathrm{q}(\mathrm{t})\right]}=\mathrm{k}_{2} \mathrm{t}-\frac{1}{\mathrm{q}_{\mathrm{e}}}$

295 For desorption, $\mathrm{q}(0)=\mathrm{q}_{0}$

$296 \quad 61: \frac{-1}{\left[\mathrm{q}_{\mathrm{e}}-q_{0}\right]}=\mathrm{C}$

297

62: $\frac{-1}{\left[\mathrm{q}_{\mathrm{e}}-\mathrm{q}(\mathrm{t})\right]}=\mathrm{k}_{2} \mathrm{t}-\frac{-1}{\left[\mathrm{q}_{\mathrm{e}}-\mathrm{q}_{0}\right]}$ 